\title{
Inference in alpha rhythm phase and amplitude modeled on Markov random field using belief propagation from electroencephalograms
}

\author{
Yasushi Naruse* \\ Kobe Advanced ICT Research Center, National Institute of Information and Communications Technology, Kobe, Hyogo 651-2492, Japan
}

Ken Takiyama

Graduate School of Frontier Sciences, The University of Tokyo, Kashiwa, Chiba 277-8561, Japan

Masato Okada

Graduate School of Frontier Sciences, The University of Tokyo, Kashiwa, Chiba 277-8561, Japan and RIKEN Brain Science Institute, Wako, Saitama 351-0198, Japan

Tsutomu Murata

Kobe Advanced ICT Research Center, National Institute of Information and Communications Technology, Kobe, Hyogo 651-2492, Japan

(Received 16 March 2010; revised manuscript received 9 June 2010; published 19 July 2010)

\begin{abstract}
Alpha rhythm is a major component of spontaneous electroencephalographic (EEG) data. We develop a novel method that can be used to estimate the instantaneous phases and amplitudes of the alpha rhythm with high accuracy by modeling the alpha rhythm phase and amplitude as Markov random field (MRF) models. By using a belief propagation technique, we construct an exact-inference algorithm that can be used to estimate instantaneous phases and amplitudes and calculate the marginal likelihood. Maximizing the marginal likelihood enables us to estimate the hyperparameters on the basis of type-II maximum likelihood estimation. We prove that the instantaneous phase and amplitude estimation by our method is consistent with that by the Hilbert transform, which has been commonly used to estimate instantaneous phases and amplitudes, of a signal filtered from observed data in the limited case that the observed data consist of only one frequency signal whose amplitude is constant and a Gaussian noise. Comparison of the performances of observation noise reduction by our method and by a Gaussian MRF model of alpha rhythm signal indicates that our method reduces observation noise more efficiently. Moreover, the instantaneous phase and amplitude estimates obtained using our method are more accurate than those obtained by the Hilbert transform. Application of our method to experimental EEG data also demonstrates that the relationship between the alpha rhythm phase and the reaction time emerges more clearly by using our method than the Hilbert transform. This indicates our method's practical usefulness. Therefore, applying our method to experimental EEG data will enable us to estimate the instantaneous phases and amplitudes of the alpha rhythm more precisely.
\end{abstract}

DOI: 10.1103/PhysRevE.82.011912

PACS number(s): $87.19 .1 \mathrm{e}, 87.85 . \mathrm{Ng}$

\section{INTRODUCTION}

The posterior $8-13 \mathrm{~Hz}$ oscillation, or the alpha rhythm, is a major component of spontaneous electroencephalographic (EEG) data [1-3]. Recent experimental studies have shown the influence of the alpha rhythm amplitude on a variety of cognitive task performances such as memory task performances $[4,5]$ and visual discrimination task performance [6]. Moreover, experimental studies have shown not only the relationship between the alpha rhythm phase and reaction time (RT) [7] that represents the visuomotor processing time but also the relationship between the alpha rhythm phase and visual awareness of light flashes at an individual luminance threshold [8] and of visual targets in a backward masking paradigm [9]. Thus the alpha rhythm has recently gained attention in neuroscientific studies [3]. In addition, brain machine interfaces (BMIs), in which modulations of spontaneous EEG activities in the alpha frequency range are used, have for some time attracted attention [10-12].

\footnotetext{
*y_naruse@po.nict.go.jp
}

Previous studies have used the Hilbert transform [13-15] or Wavelet transform $[16,17]$ to estimate instantaneous phases and amplitudes of the alpha rhythm. However, observed EEG data contain not only neural signals but also observation noises; the noises cause loss of accuracy of the instantaneous phases and amplitudes estimated by these methods in a single trial. To increase the signal-to-noise ratio, the data are averaged over many trials. However, recording of many trials exhausts subjects; thus it is important to estimate instantaneous phases and amplitudes of the alpha rhythm with a high degree of accuracy to reduce the number of trials for averaging. Also, averaging suppresses variable neural activities between trials. Since the alpha rhythm phase and amplitude vary between trials $[18,19]$, it is useful if we can extract reliable information from a single trial. The BMIs analyze single-trial EEG data to extract neural signal; therefore the greater the accuracy of the estimated instantaneous phases and amplitudes, the greater is the improvement in the BMIs' abilities. Accuracy enhancement of the estimated instantaneous phases and amplitudes can contribute to advancing EEG-based neuroscientific studies and BMI techniques. 
In this study, we develop a novel method to estimate instantaneous phases and amplitudes of the alpha rhythm with a high degree of accuracy by modeling the alpha rhythm phase and amplitude as Markov random field (MRF) models. Observed data consist of the alpha rhythm and observation noises. A Gaussian MRF model is commonly used for image restoration [20-22], and hence, we can construct a Gaussian MRF model of the signal (MRFS) of the alpha rhythm so as to reduce the observation noise. However, the alpha rhythm consists of phase and amplitude of a particular frequency. Thus, we can also construct MRF models of the phase and amplitude (MRFPA) of the alpha rhythm by separating the phase and amplitude from an alpha rhythm signal.

The phase of the alpha rhythm advances with some fluctuation around an individual alpha frequency, and the amplitude smoothly fluctuates over time [23]. Accordingly, we construct the MRFPA of the alpha rhythm by introducing $a$ priori knowledge about the alpha rhythm. However, the amount of phase fluctuation, the smoothness of amplitude fluctuation, and the individual alpha frequency are different between subjects, and the amount of observation noise is different under different experimental conditions. Therefore, the hyperparameters, which control the amount of observation noise, the amount of phase fluctuation, the smoothness of amplitude fluctuation, and the individual alpha frequency, also must be estimated.

We can apply belief propagation (BP), which is one of statistical inference techniques [24], to MRF models $[21,25,26]$. By using BP, we formulate an exact-inference algorithm that can estimate instantaneous phases and amplitudes, and calculate a marginal likelihood of the MRFPA. Maximizing the marginal likelihood enables us to estimate the hyperparameters based on type-II maximum likelihood estimation [22].

In order to promote better understanding of the MRFPA, we investigate the relationship between the Hilbert transform and the MRFPA, and we prove that the instantaneous phase and amplitude estimation by the MRFPA is consistent with that by the Hilbert transform of a signal filtered from observed data, in the case that the observed data consist of only one frequency signal whose amplitude is constant and a Gaussian noise.

We numerically estimate the instantaneous phase and amplitude of simulated EEG data by the MRFPA using the BP and investigate the performance of the MRFPA with regard to observation noise reduction and accuracy of the instantaneous phase and amplitude estimation. By comparing the performances of observation noise reduction by the MRFPA and MRFS, we demonstrate the importance of introducing the MRFPA. Next, we show that the instantaneous phases and amplitudes estimated by the MRFPA are more accurate than those estimated by the Hilbert transform. Then we show practical usefulness of the MRFPA for experimental EEG data, demonstrating that the relationship between the alpha rhythm phase and the RT emerges more clearly by using the MRFPA than the Hilbert transform.

\section{MODEL AND ALGORITHM}

In this section, we define an MRFPA and construct an exact-inference algorithm for estimating instantaneous phases and amplitudes by the MRFPA using the BP. Then, we present the definition and algorithm of an MRFS and the algorithm of the Hilbert transform.

\section{A. Markov random field models of phase and amplitude (MRFPA)}

\section{Definition of MRFPA}

The observed data of the alpha rhythm at the sampling point $k$ is denoted by $y_{k} \cdot y_{k}$ includes an independent observation noise between sampling points and is thus expressed as

$$
y_{k}=a_{k} \cos \left(x_{k}\right)+\xi,
$$

where $x_{k}$ and $a_{k}$ denote the instantaneous phase and amplitude of the alpha rhythm at the sampling point $k$, respectively, and $\xi$ denotes the observation noise. Here, we assume the noise as a Gaussian noise, and therefore, the likelihood function is

$$
p\left(y_{k} \mid a_{k}, x_{k}, \alpha\right)=\sqrt{\frac{\alpha}{2 \pi}} \exp \left\{-\frac{\alpha}{2}\left[y_{k}-a_{k} \cos \left(x_{k}\right)\right]^{2}\right\},
$$

where $\alpha$ is the hyperparameter that controls the observation noise strength. Since the frequency of the alpha rhythm fluctuates around an individual alpha frequency and its amplitude fluctuates smoothly [23], we assume that the phase, $\boldsymbol{x}$ $=\left\{x_{1}, x_{2}, \ldots, x_{N}\right\}$, smoothly advances with some fluctuation around the individual alpha frequency and the amplitude, $\boldsymbol{a}$ $=\left\{a_{1}, a_{2}, \ldots, a_{N}\right\}$, smoothly fluctuates over time. $N$ indicates the number of sampling points. Note that the domain of the phase is $[0,2 \pi)$ and that of the amplitude is $[0, \infty)$. Thus, we define the prior distribution of the phase based on the vonMises distribution as

$$
p(\boldsymbol{x} \mid \beta, \omega)=\frac{1}{Z_{\mathrm{p}}(\beta)} \exp \left[\sum_{n=1}^{N-1} \beta \cos \left(x_{n+1}-x_{n}-\omega\right)\right],
$$

where $Z_{\mathrm{p}}(\beta)$ indicates the normalization constant of $p(\boldsymbol{x} \mid \beta, \omega)$, and we define the prior distribution of the amplitude as

$$
p(\boldsymbol{a} \mid \gamma)=\frac{1}{Z_{\mathrm{a}}(\gamma)} \exp \left[-\sum_{n=1}^{N-1} \frac{\gamma}{2}\left(a_{n+1}-a_{n}\right)^{2}\right],
$$

where $Z_{\mathrm{a}}(\gamma)$ denotes the normalization constant of $p(\boldsymbol{a} \mid \gamma)$. The hyperparameters $\beta, \gamma$, and $\omega$ control the amount of phase fluctuation, the smoothness of amplitude fluctuation, and individual alpha frequency, respectively. Assuming that the prior distributions of the phase and amplitude are independent, we can express the joint distribution as

$$
p(\boldsymbol{y}, \boldsymbol{a}, \boldsymbol{x} \mid \alpha, \beta, \gamma, \omega)=p(\boldsymbol{y} \mid \boldsymbol{a}, \boldsymbol{x}, \alpha) p(\boldsymbol{a} \mid \gamma) p(\boldsymbol{x} \mid \beta, \omega) .
$$

Thus, the posterior distribution is

$$
p(\boldsymbol{a}, \boldsymbol{x} \mid \boldsymbol{y}, \alpha, \beta, \gamma, \omega)=\frac{p(\boldsymbol{y} \mid \boldsymbol{a}, \boldsymbol{x}, \alpha) p(\boldsymbol{a} \mid \gamma) p(\boldsymbol{x} \mid \beta, \omega)}{Z(\alpha, \beta, \gamma, \omega)},
$$

where $Z(\alpha, \beta, \gamma, \omega)$ represents the normalization constant of $p(\boldsymbol{a}, \boldsymbol{x} \mid \boldsymbol{y}, \alpha, \beta, \gamma, \omega)$ and is expressed as 


$$
Z(\alpha, \beta, \gamma, \omega)=p(y \mid \alpha, \beta, \gamma, \omega) .
$$

\section{Belief propagation algorithm for estimating instantaneous phase and amplitude}

We formulate a BP algorithm for estimating instantaneous phases, amplitudes, and hyperparameters. First we estimate the hyperparameters on the basis of type-II maximum likelihood estimation and then we estimate instantaneous phases and amplitudes by the maximizer of posterior marginal (MPM) estimation.

Marginalization of $\boldsymbol{a}$ and $\boldsymbol{x}$ in Eq. (5) gives the marginal likelihood by

$$
\begin{aligned}
p(\boldsymbol{y} \mid \alpha, \beta, \gamma, \omega)= & Z(\alpha, \beta, \gamma, \omega) \\
= & \int_{0}^{\infty} \prod_{n=1}^{N} d a_{n} \int_{0}^{2 \pi} \prod_{n=1}^{N} d x_{n} \\
& \times p(\boldsymbol{y} \mid \boldsymbol{a}, \boldsymbol{x}, \alpha) p(\boldsymbol{a} \mid \gamma) p(\boldsymbol{x} \mid \beta, \omega) \\
= & \int_{0}^{\infty} \prod_{n=1}^{N} d a_{n} \int_{0}^{2 \pi} \prod_{n=1}^{N} d x_{n} \prod_{n=1}^{N} p\left(y_{n} \mid a_{n}, x_{n}, \alpha\right) \\
& \times \frac{1}{Z_{\mathrm{p}}(\beta)} \exp \left[\sum_{n=1}^{N-1} \beta \cos \left(x_{n+1}-x_{n}-\omega\right)\right] \\
& \times \frac{1}{Z_{\mathrm{a}}(\gamma)} \exp \left[-\sum_{n=1}^{N-1} \frac{\gamma}{2}\left(a_{n+1}-a_{n}\right)^{2}\right] .
\end{aligned}
$$

The marginalization in Eq. (8) involves computational difficulty since it includes $2 N$ integrations. The order of the computational complexity is $\mathcal{O}\left(n_{\mathrm{p}}^{N} \times n_{\mathrm{a}}^{N}\right)$, where $n_{\mathrm{p}}$ and $n_{\mathrm{a}}$ represent the partition numbers in the numerical integration for the phase and amplitude, respectively. However, by using the BP algorithm with the recurrence formulas of messages, $M_{L}$ and $M_{R}$, we can marginalize it in the order $\mathcal{O}\left(N \times n_{\mathrm{p}}^{2} \times n_{\mathrm{a}}^{2}\right)$. Here, the definitions of the messages are

$$
\begin{aligned}
M_{L}\left(a_{k}, x_{k} \mid \alpha, \beta, \gamma, \omega\right)= & \int_{0}^{\infty} d a_{k-1} \int_{0}^{2 \pi} d x_{k-1} p\left(y_{k-1} \mid a_{k-1}, x_{k-1}, \alpha\right) \\
& \times \mu\left(a_{k}, a_{k-1}, \gamma\right) \nu\left(x_{k}, x_{k-1}, \beta, \omega\right) \\
& \times M_{L}\left(a_{k-1}, x_{k-1} \mid \alpha, \beta, \gamma, \omega\right),
\end{aligned}
$$

and

$$
\begin{aligned}
M_{R}\left(a_{k}, x_{k} \mid \alpha, \beta, \gamma, \omega\right)= & \int_{0}^{\infty} d a_{k+1} \int_{0}^{2 \pi} d x_{k+1} p\left(y_{k+1} \mid a_{k+1}, x_{k+1}, \alpha\right) \\
& \times \mu\left(a_{k+1}, a_{k}, \gamma\right) \nu\left(x_{k+1}, x_{k}, \beta, \omega\right) \\
& \times M_{R}\left(a_{k+1}, x_{k+1} \mid \alpha, \beta, \gamma, \omega\right),
\end{aligned}
$$

where

$$
\mu\left(a_{k+1}, a_{k}, \gamma\right)=\exp \left[-\frac{\gamma}{2}\left(a_{k+1}-a_{k}\right)^{2}\right]
$$

and

$$
\nu\left(x_{k+1}, x_{k}, \beta, \omega\right)=\exp \left[\beta \cos \left(x_{k+1}-x_{k}-\omega\right)\right] .
$$

Since we do not have the previous observed data $\left(y_{0}\right)$ and next observed data $\left(y_{N+1}\right)$ for the observed data set $\left(\boldsymbol{y}=\left\{y_{1}, y_{2}, \ldots, y_{N}\right\}\right)$, we have no a priori knowledge about the initial messages $M_{L}\left(a_{1}, x_{1} \mid \alpha, \beta, \gamma, \omega\right)$ and $M_{R}\left(a_{N}, x_{N} \mid \alpha, \beta, \gamma, \omega\right)$. Therefore, we set the value of the initial messages as one regardless of the values of the phase and amplitude since any values of phase and amplitude can occur in equal probability, and the initial messages are expressed as

$$
M_{L}\left(a_{1}, x_{1} \mid \alpha, \beta, \gamma, \omega\right)=1,
$$

and

$$
M_{R}\left(a_{N}, x_{N} \mid \alpha, \beta, \gamma, \omega\right)=1 .
$$

Thus, the marginal likelihood is given by

$$
p(\boldsymbol{y} \mid \alpha, \beta, \gamma, \omega)=Z(\alpha, \beta, \gamma, \omega)=\int_{0}^{\infty} d a_{k} \int_{0}^{2 \pi} d x_{k} \frac{p\left(y_{k} \mid a_{k}, x_{k}, \alpha\right) M_{L}\left(a_{k}, x_{k} \mid \alpha, \beta, \gamma, \omega\right) M_{R}\left(a_{k}, x_{k} \mid \alpha, \beta, \gamma, \omega\right)}{Z_{\mathrm{p}}(\beta) Z_{\mathrm{a}}(\gamma)} .
$$

On the basis of type-II maximum likelihood estimation, the estimation values of the hyperparameters, $\hat{\alpha}, \hat{\beta}, \hat{\gamma}$, and $\hat{\omega}$, are

$$
\{\hat{\alpha}, \hat{\beta}, \hat{\gamma}, \hat{\omega}\}=\arg \max _{\{\alpha, \beta, \gamma, \omega\}} Z(\alpha, \beta, \gamma, \omega) .
$$

In the case that we have more than one trial, it is plausible to use all the trials for estimating the hyperparameters. Here we express the marginal likelihood of the $h$ th trial as $Z_{h}(\alpha, \beta, \gamma, \omega)$. In this case, the estimation values of the hyperparameters, $\hat{\alpha}, \hat{\beta}, \hat{\gamma}$, and $\hat{\omega}$ are

$$
\{\hat{\alpha}, \hat{\beta}, \hat{\gamma}, \hat{\omega}\}=\arg \max _{\{\alpha, \beta, \gamma, \omega\}} \prod_{h=1}^{H} Z_{h}(\alpha, \beta, \gamma, \omega),
$$

where $H$ indicates the number of trials. In this study, we estimated the hyperparameters using Eq. (17). To estimate the hyperparameters based on Eq. (17), we used interiorpoint algorithm with optimization toolbox in MATLAB (The MathWorks).

Next, marginal posterior distribution gives the instantaneous phase estimation value $\hat{x}_{k}$ and the instantaneous amplitude estimation value $\hat{a}_{k}$ by the following MPM: 


$$
\left\{\hat{a}_{k}, \hat{x}_{k}\right\}=\arg \max _{\left\{a_{k}, x_{k}\right\}} p\left(a_{k}, x_{k} \mid \boldsymbol{y}, \alpha, \beta, \gamma, \omega\right),
$$

where the marginal posterior distribution is given by

$$
\begin{aligned}
p\left(a_{k}, x_{k} \mid \boldsymbol{y}, \alpha, \beta, \gamma, \omega\right)= & \frac{p\left(y_{k} \mid a_{k}, x_{k}, \alpha\right)}{Z(\alpha, \beta, \gamma, \omega) Z_{\mathrm{p}}(\beta) Z_{\mathrm{a}}(\gamma)} \\
& \times M_{L}\left(a_{k}, x_{k} \mid \alpha, \beta, \gamma, \omega\right) \\
& \times M_{R}\left(a_{k}, x_{k} \mid \alpha, \beta, \gamma, \omega\right) .
\end{aligned}
$$

\section{B. Markov random field model of signal (MRFS)}

\section{Definition of MRFS}

The observed data $y_{k}$ can be expressed as

$$
y_{k}=m_{k}+\xi,
$$

where $m_{k}$ represents the signal of the alpha rhythm at the sampling point $k$, and $\xi$ denotes an independent observation noise between these sampling points. Note that the domain of the signal is $(-\infty, \infty)$. Here, we assume the noise to be Gaussian, and hence, the likelihood function is

$$
p\left(y_{k} \mid m_{k}, \eta\right)=\sqrt{\frac{\eta}{2 \pi}} \exp \left[-\frac{\eta}{2}\left(y_{k}-m_{k}\right)^{2}\right],
$$

where $\eta$ is the hyperparameter that controls the observation noise strength. The prior distribution of the signal based on a Gaussian MRF model is

$$
p(\boldsymbol{m} \mid \lambda)=\frac{1}{Z_{\mathrm{s}}(\lambda)} \exp \left[-\sum_{n=1}^{N-1} \frac{\lambda}{2}\left(m_{n+1}-m_{n}\right)^{2}\right],
$$

where $\lambda$ is the hyperparameter that control the smoothness of the signal, and $Z_{\mathrm{s}}(\lambda)$ is the normalization constant of $p(\boldsymbol{m} \mid \lambda)$.

The joint distribution is

$$
p(\boldsymbol{y}, \boldsymbol{m} \mid \eta, \lambda)=p(\boldsymbol{y} \mid \boldsymbol{m}, \eta) p(\boldsymbol{m} \mid \lambda) .
$$

Thus, the posterior distribution is

$$
p(\boldsymbol{m} \mid \boldsymbol{y}, \eta, \lambda)=\frac{p(\boldsymbol{y} \mid \boldsymbol{m}, \eta, \lambda) p(\boldsymbol{m} \mid \lambda)}{Z(\eta, \lambda)},
$$

where

$$
Z(\eta, \lambda)=p(\boldsymbol{y} \mid \eta, \lambda) .
$$

\section{Belief propagation algorithm for estimating signal}

Here we define the messages as

$$
\begin{aligned}
M_{L}^{*}\left(m_{k} \mid \eta, \lambda\right)= & \int_{-\infty}^{\infty} d m_{k-1} p\left(y_{k-1} \mid m_{k-1}, \eta\right) \rho\left(m_{k}, m_{k-1}, \lambda\right) \\
& \times M_{L}^{*}\left(m_{k-1} \mid \eta, \lambda\right),
\end{aligned}
$$

and

$$
\begin{aligned}
M_{R}^{*}\left(m_{k} \mid \eta, \lambda\right)= & \int_{-\infty}^{\infty} d m_{k+1} p\left(y_{k+1} \mid m_{k+1}, \eta\right) \\
& \times \rho\left(m_{k}, m_{k+1}, \lambda\right) M_{R}^{*}\left(m_{k+1} \mid \eta, \lambda\right),
\end{aligned}
$$

where

$$
\rho\left(m_{k}, m_{k+1}, \lambda\right)=\exp \left[-\frac{\lambda}{2}\left(m_{k+1}-m_{k}\right)^{2}\right] .
$$

The initial values of the messages are defined as

$$
M_{L}^{*}\left(m_{1} \mid \eta, \lambda\right)=1,
$$

and

$$
M_{R}^{*}\left(m_{N} \mid \eta, \lambda\right)=1 .
$$

The marginal likelihood is given by

$$
\begin{aligned}
p(\boldsymbol{y} \mid \eta, \lambda) & =Z(\eta, \lambda) \\
& =\int_{-\infty}^{\infty} d m_{k} \frac{p\left(y_{k} \mid m_{k}, \eta\right) M_{L}^{*}\left(m_{k} \mid \eta, \lambda\right) M_{R}^{*}\left(m_{k} \mid \eta, \lambda\right)}{Z_{\mathrm{s}}(\lambda)} .
\end{aligned}
$$

Here, we express the marginal likelihood of the $h$ th trial as $Z_{h}(\eta, \lambda)$. The estimation values of the hyperparameters, $\hat{\eta}$ and $\hat{\lambda}$, on the basis of type-II maximum likelihood estimation are

$$
\{\hat{\eta}, \hat{\lambda}\}=\arg \max _{\{\eta, \lambda\}} \prod_{h=1}^{H} Z_{h}(\eta, \lambda) .
$$

The marginal posterior distribution of $m_{k}$ is given by

$$
p\left(m_{k} \mid \boldsymbol{y}\right)=\frac{p\left(y_{k} \mid m_{k}\right) M_{L}^{*}\left(m_{k}\right) M_{R}^{*}\left(m_{k}\right)}{Z(\eta, \lambda) Z_{\mathrm{s}}(\lambda)} .
$$

By the MPM that is

$$
\hat{m}_{k}=\arg \max _{m_{k}} \frac{p\left(y_{k} \mid m_{k}, \eta\right) M_{L}^{*}\left(m_{k} \mid \eta, \lambda\right) M_{R}^{*}\left(m_{k} \mid \eta, \lambda\right)}{Z(\eta, \lambda) Z_{\mathrm{s}}(\lambda)},
$$

we estimate the magnitude $\hat{m}_{k}$.

The MRFS can be solved analytically [27]. The analytical solution of the MRFS is shown in the Appendix. The MRFS is known to be consistent with the Wiener filter [28]. Note that the MRFS estimates signals and not amplitudes and phases as in the MRFPA.

\section{Hilbert transform}

The Hilbert transform is commonly used to estimate instantaneous phases and amplitudes of the alpha rhythm whose frequency fluctuates [13-15]. With $y_{k}^{\mathrm{r}}(k \in \mathcal{Z}[1, N])$ denoting a time course and $Y_{l}^{\mathrm{r}}(l \in \mathcal{Z}[0, N-1])$ its Fourier transform, we define $Y_{l}^{\mathrm{i}}$ as

$$
Y_{l}^{\mathrm{i}}=H_{l} Y_{l}^{\mathrm{r}},
$$

where

$$
H_{l}= \begin{cases}-j, & 0<l \leq(N-1) / 2 \\ j, & (N-1) / 2<l \leq N-1 \\ 0, & \text { otherwise },\end{cases}
$$

when $N$ is odd, and 


$$
H_{l}= \begin{cases}-j, & 0<l<N / 2 \\ j, & N / 2<l \leq N-1 \\ 0, & \text { otherwise }\end{cases}
$$

when $N$ is even. Then, we obtain a complex time course $y_{k}^{\mathrm{r}}$ $+j y_{k}^{\mathrm{i}}$, which is the inverse Fourier transform of $Y_{l}^{\mathrm{r}}+j Y_{l}^{\mathrm{i}}$, and $y_{k}^{\mathrm{r}}+j y_{k}^{\mathrm{i}}$ is the Hilbert transform of $y_{k}^{\mathrm{r}}$. Because $y_{k}^{\mathrm{r}}+j y_{k}^{\mathrm{i}}$ can be expressed as $\widetilde{a}_{k} \exp \left(j \widetilde{x}_{k}\right)$, the instantaneous phase and amplitude of $y_{k}^{\mathrm{r}}$ estimated by the Hilbert transform are $\widetilde{x}_{k}$ and $\widetilde{a}_{k}$, respectively [29].

Further, when $N$ is odd,

$$
Y_{l}=Y_{l}^{\mathrm{r}}+j Y_{l}^{\mathrm{i}}= \begin{cases}Y_{l}^{\mathrm{r}}, & l=0 \\ 2 Y_{l}^{\mathrm{r}}, & 0<l \leq(N-1) / 2 \\ 0, & (N-1) / 2<l \leq N-1 .\end{cases}
$$

$y_{k}^{\mathrm{r}}$, which is the original time course, is the real part of the inverse Fourier transform of $Y_{l}$, and is hence expressed as

$$
\begin{aligned}
y_{k}^{\mathrm{r}} & =\operatorname{Re}\left\{\sum_{l=0}^{N-1} Y_{l} \exp \left[\frac{j 2 \pi(k-1) l}{N}\right]\right\} \\
& =\sum_{l=0}^{(N-1) / 2}\left|Y_{l}\right| \cos \left[\frac{2 \pi(k-1) l}{N}+\arg \left(Y_{l}\right)\right] .
\end{aligned}
$$

Thus, we can express $y_{k}^{\mathrm{r}}$ by the Fourier series, using $Y_{l}$. In the case that $N$ is even, we can also express $y_{k}^{\mathrm{r}}$ by the Fourier series, using $Y_{l}$.

\section{RELATIONSHIP BETWEEN THE HILBERT TRANSFORM AND THE MARKOV RANDOM FIELD MODELS OF PHASE AND AMPLITUDE}

In this section, we clarify the relationship between the Hilbert transform and the MRFPA. Here, we assume that observed data consist of only one frequency signal whose amplitude is constant and a Gaussian noise. In this case, the instantaneous phase and amplitude are determined by their respective values at the previous sampling point, and thus, we can modify the MRFPA as follows: We obtain the prior distribution of the phase as

$$
p(\boldsymbol{x} \mid \omega)=\prod_{n=1}^{N-1} \delta\left(x_{n+1}-x_{n}-\omega\right),
$$

where $\delta(x)$ denotes the Dirac delta function; the prior distribution of the amplitude is obtained as

$$
p(\boldsymbol{a})=\prod_{n=1}^{N-1} \delta\left(a_{n+1}-a_{n}\right) .
$$

The likelihood function is

$$
p\left(y_{k} \mid a_{k}, x_{k}, \alpha\right)=\sqrt{\frac{\alpha}{2 \pi}} \exp \left\{-\frac{\alpha}{2}\left[y_{k}-a_{k} \cos \left(x_{k}\right)\right]^{2}\right\} .
$$

The posterior distribution is given by

$$
p(\boldsymbol{a}, \boldsymbol{x} \mid \boldsymbol{y}, \alpha, \omega)=\frac{p(\boldsymbol{y} \mid \boldsymbol{a}, \boldsymbol{x}, \alpha) p(\boldsymbol{a}) p(\boldsymbol{x} \mid \omega)}{Z(\alpha, \omega)},
$$

where $Z(\alpha, \omega)$ is the normalization constant of $p(\boldsymbol{a}, \boldsymbol{x} \mid \boldsymbol{y}, \alpha, \omega)$. by

The marginal posterior distribution of $x_{1}$ and $a_{1}$ is given

$$
\begin{aligned}
p\left(a_{1}, x_{1} \mid \boldsymbol{y}, \alpha, \omega\right)= & \frac{1}{Z(\alpha, \omega)} \int_{0}^{\infty} \prod_{n=2}^{N} d a_{n} \int_{0}^{2 \pi} \prod_{n=2}^{N} \\
& \times d x_{n} p(\boldsymbol{y} \mid \boldsymbol{a}, \boldsymbol{x}, \alpha) p(\boldsymbol{a}) p(\boldsymbol{x} \mid \omega) \\
= & \frac{1}{Z(\alpha, \omega)} \sqrt{\frac{\alpha}{2 \pi}} N \exp \left(-\frac{\alpha}{2}\right. \\
& \left.\times\left(\sum_{n=1}^{N}\left\{y_{n}-a_{1} \cos \left[x_{1}+\omega(n-1)\right]\right\}^{2}\right)\right) .
\end{aligned}
$$

The marginal posterior distribution gives the instantaneous phase estimation value $\hat{x}_{1}$ and amplitude estimation value $\hat{a}_{1}$ by the following MPM:

$$
\left\{\hat{a}_{1}, \hat{x}_{1}\right\}=\arg \max _{\left\{a_{1}, x_{1}\right\}} p\left(a_{1}, x_{1} \mid \boldsymbol{y}, \alpha, \omega\right) .
$$

Here, we define $Q$ as

$$
Q=\min _{\left(a_{1}, x_{1}\right)}\left(\sum_{n=1}^{N}\left\{y_{n}-a_{1} \cos \left[x_{1}+\omega(n-1)\right]\right\}^{2}\right),
$$

and $a_{1}$ and $x_{1}$ that realize $Q$ are consistent with $\hat{a}_{1}$ and $\hat{x}_{1}$ estimated by the MPM. From Eq. (39), $y_{k}$ can be expressed as

$$
y_{k}=\sum_{l=0}^{(N-1) / 2}\left|Y_{l}\right| \cos \left[\frac{2 \pi(k-1) l}{N}+\arg \left(Y_{l}\right)\right],
$$

where $Y_{l}$ is obtained from Eq. (38). By substituting Eq. (47) into Eq. (46), we can express $Q$ as

$$
\begin{aligned}
Q= & \min _{\left(a_{1}, x_{1}\right)}\left(\sum _ { n = 1 } ^ { N } \left\{\sum_{l=0}^{(N-1) / 2}\left|Y_{l}\right| \cos \left[\frac{2 \pi(n-1) l}{N}+\arg \left(Y_{l}\right)\right]\right.\right. \\
& \left.\left.-a_{1} \cos \left[\omega(n-1)+x_{1}\right]\right\}^{2}\right) .
\end{aligned}
$$

Here, the time courses,

$$
\begin{aligned}
& \cos \left(\frac{2 \pi(k-1)+\arg \left(Y_{1}\right)}{N}\right), \cos \left(\frac{4 \pi(k-1)+\arg \left(Y_{2}\right)}{N}\right), \cdots, \\
& \cos \left(\frac{2 \pi(k-1)(N-1) / 2+\arg \left(Y_{(N-1) / 2}\right)}{N}\right),
\end{aligned}
$$

are orthogonal to each other because of the orthogonality property of Fourier series, and therefore, $a_{1}=\left|Y_{\psi}\right|$ and $x_{1}$ $=\arg \left(Y_{\psi}\right)$, where $l=\psi=\frac{\omega}{2 \pi} N$, realize $Q$. Thus, the estimated instantaneous phase $\hat{x}_{k}$ and amplitude $\hat{a}_{k}$ of $y_{k}$ by this modified MRFPA model are 


$$
\hat{x}_{k}=\frac{2 \pi(k-1) \psi}{N}+\arg \left(Y_{\psi}\right),
$$

and

$$
\hat{a}_{k}=\left|Y_{\psi}\right|,
$$

respectively.

Next, we filter $y_{k}$ to exclude all the frequencies other than $\psi$, then, the filtered signal $y_{k}^{\psi}$ is expressed as

$$
y_{k}^{\psi}=\left|Y_{\psi}\right| \cos \left(\frac{2 \pi(k-1) \psi}{N}+\arg \left(Y_{\psi}\right)\right) \text {. }
$$

Thus, the instantaneous phase $\tilde{x}_{k}^{\psi}$ and amplitude $\widetilde{a}_{k}^{\psi}$ of $y_{k}^{\psi}$ estimated by the Hilbert transform are

$$
\tilde{x}_{k}^{\psi}=\frac{2 \pi(k-1) \psi}{N}+\arg \left(Y_{\psi}\right),
$$

and

$$
\widetilde{a}_{k}^{\psi}=\left|Y_{\psi}\right|,
$$

respectively, and they correspond to the estimated instantaneous phase and amplitude by the modified MRFPA model [Eqs. (49) and (50)].

Therefore, when the observed data consist of only one frequency signal with constant amplitude and Gaussian noise, the instantaneous phase and amplitude estimated by the modified MRFPA model are consistent with those estimated by the Hilbert transform of the filtered signal. In fact, the amplitude and frequency of the alpha rhythm fluctuate, and hence, it is reasonable to advance the modified MRFPA model, which is comparable to the Hilbert transform, according to a priori knowledge about the alpha rhythm and derive the MRFPA.

\section{RESULTS}

\section{A. Numerical experiments}

Instantaneous phases $\boldsymbol{x}$, instantaneous amplitudes $\boldsymbol{a}$, and simulated data $\boldsymbol{y}$ were generated on the basis of $N=300, \alpha$ $=0.5, \beta=5, \gamma=1$, and $\omega=0.6$. By repeating this generation process, we generated 15 trials. Figure 1(a) shows typical time courses of simulated data and experimental EEG data. Figure 1(b) shows the Fourier spectra averaged over the 15 trials of the simulated data and the experimental EEG data. These averaged Fourier spectra are similar to each other; this result indicates that the simulated data generated on the basis of the hyperparameters are similar to the experimental EEG data. $\hat{\boldsymbol{x}}$ and $\hat{\boldsymbol{a}}$ were estimated from $\boldsymbol{y}$ by the MRFPA. Figure 2 shows a typical marginal posterior distribution for estimating $\hat{a}_{k}$ and $\hat{x}_{k}$ based on Eq. (18), indicating a bimodal distribution in which there are a large peak around $x_{k}=4 \pi / 3, a_{k}$ $=12$ and a small peak around $x_{k}=2 \pi / 3, a_{k}=12$. The reason why the marginal posterior distribution is bimodal is that the cosine function has an uncertainty, which is $\cos (\theta)=\cos (2 \pi$ $-\theta)$. In the case of a bimodal distribution, the posterior mean (PM) estimation method sometimes yields an inappropriate estimated value that lies between high-probability regions, (a)
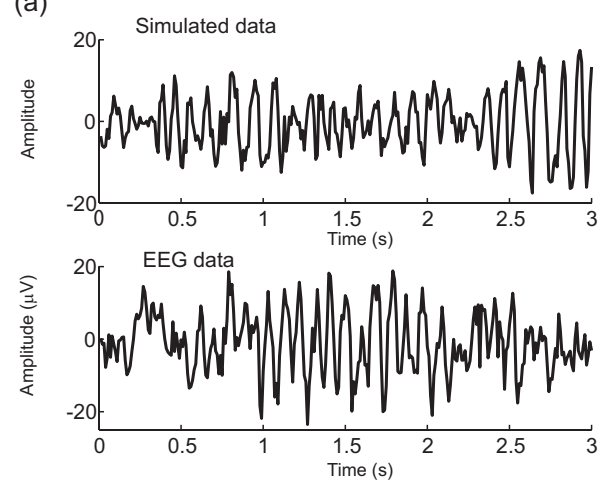

(b)

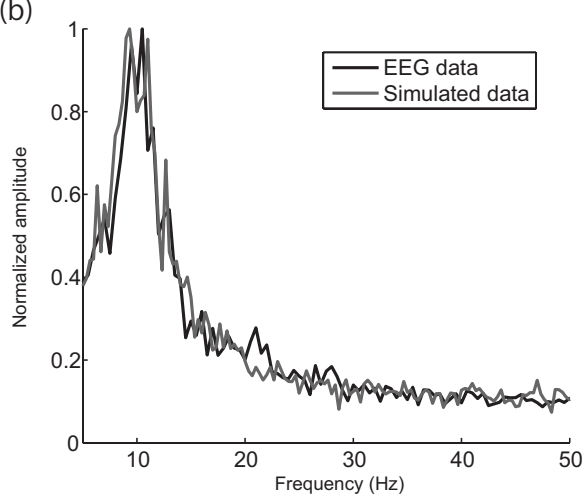

FIG. 1. (a) Typical time courses of simulated data $\boldsymbol{y}$ (upper) and experimental EEG data (lower). The simulated data were assumed to be sampled at $100 \mathrm{~Hz}$. The experimental EEG data were measured at $\mathrm{Pz}$ electrode when the subject was under eye-closed condition [19]. (b) The averaged Fourier spectra of experimental EEG data (black line) and the simulated data (gray line). The Fourier spectra of 15 trials were averaged for each data, and each averaged Fourier spectrum was normalized by the maximum amplitude within 5-50 Hz.

and hence, the MPM, which is adopted in this study, is more suitable than the PM.

In order to compare the performances of observation noise reduction by the MRFPA and the MRFS, we carried out the instantaneous phase and amplitude estimation by the

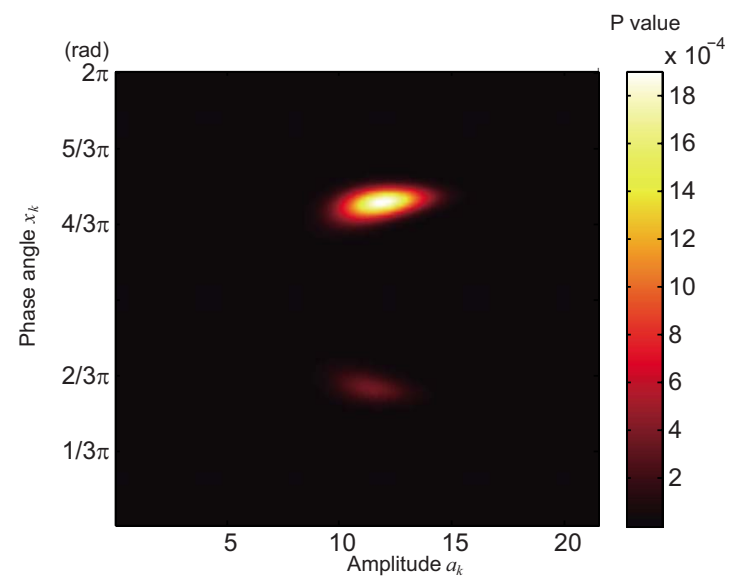

FIG. 2. (Color online) A typical marginal posterior distribution. 

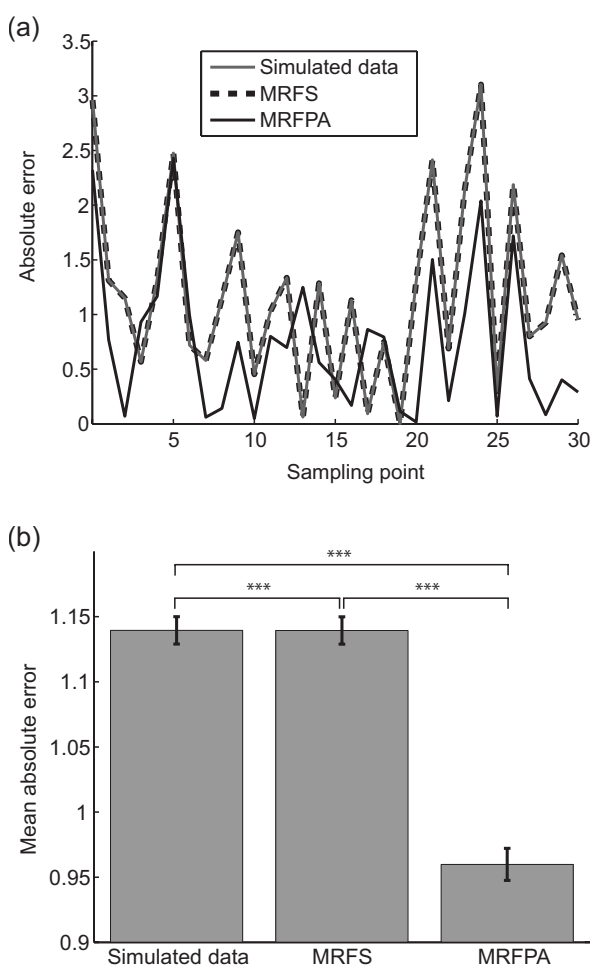

FIG. 3. (a) Typical time courses of absolute observation error of the simulated data (gray line), absolute estimate error of the MRFS (black broken line), and that of the MRFPA (black line). The time courses of absolute observation error of the simulated data and absolute estimate error of the MRFS were almost overlapped. (b) Mean absolute observation and estimate errors averaged over the trials. The error bars indicate the standard errors. $* * *$ indicates $p$ $<0.0001$

MRFPA and signal estimation by the MRFS from the simulated data of the generated 15 trials. The estimated hyperparameter values for the MRFPA were $\hat{\alpha}=0.497, \hat{\beta}=4.82, \hat{\gamma}$ $=1.24$, and $\hat{\omega}=0.604$, and those for the MRFS were $\hat{\eta}=816$ and $\hat{\lambda}=0.0336$. Figure 3 (a) shows typical time courses of absolute observation errors, which are $|\boldsymbol{y}-\boldsymbol{a} \cos (\boldsymbol{x})|$, and absolute estimation errors, which are $|\hat{\boldsymbol{a}} \cos (\hat{\boldsymbol{x}})-\boldsymbol{a} \cos (\boldsymbol{x})|$ for the MRFPA and $|\hat{\boldsymbol{m}}-\boldsymbol{a} \cos (\boldsymbol{x})|$ for the MRFS. Figure 3(b) shows the mean absolute estimate and observation errors averaged over the trials. Both the mean absolute estimate errors were significantly less than the mean absolute observation error of the simulated data (Wilcoxon signed rank test, $p$ $<0.0001)$. Note that the difference between the mean absolute estimate error of the MRFS and mean absolute observation error of the simulated data was significant in spite of the small difference, because the former was less than the latter in all the trials. However, the difference was so small that the observation noise reduction performance of the MRFS was very low. The mean absolute estimate error of the MRFPA was less than that of the MRFS in all the trials (Wilcoxon signed rank test, $p<0.0001)$. This result indicates that the observation noise can be reduced more efficiently by the MRFPA than by the MRFS.

Next, we investigated the accuracy of the instantaneous phases and amplitudes estimated by the MRFPA. We compared the mean absolute estimate errors of instantaneous
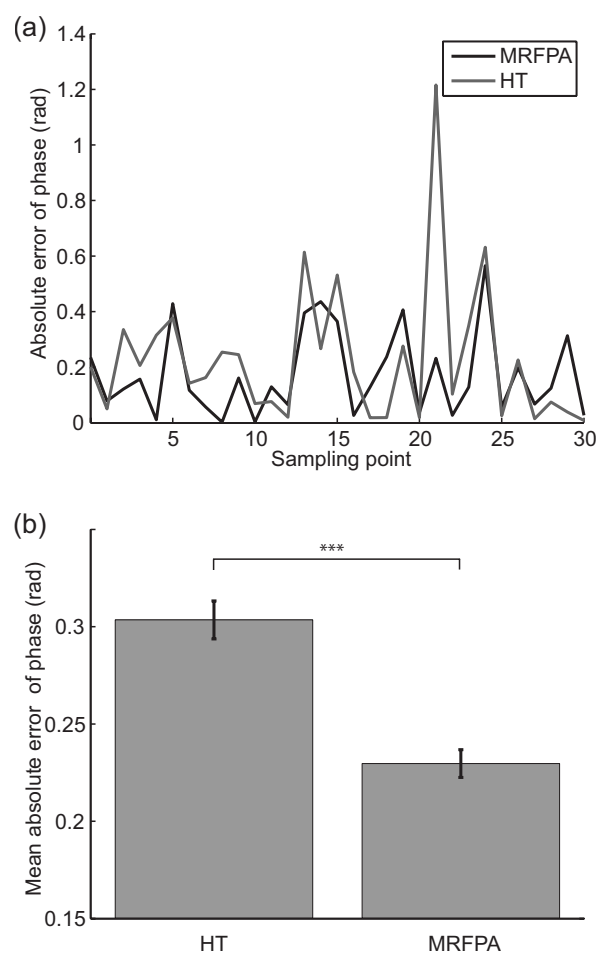

FIG. 4. (a) Typical time courses of absolute estimate error of instantaneous phase estimated by the MRFPA (black line) and the Hilbert transform (gray line). (b) Mean absolute estimate errors of instantaneous phases. Values averaged over the trials are illustrated. The error bars indicate the standard errors. $* * *$ indicates $p$ $<0.0001$.

phases and amplitudes estimated by the MRFPA with those estimated by the Hilbert transform. Since the phase has a periodic boundary condition, the mean absolute error of phases is defined as

$$
\begin{gathered}
S e=\sum_{n=1}^{N} z_{n} / N, \\
z_{n}=\min \left(\left|x_{n}-\bar{x}_{n}\right|,\left|x_{n}-\bar{x}_{n}+2 \pi\right|,\left|x_{n}-\bar{x}_{n}-2 \pi\right|\right),
\end{gathered}
$$

where $\bar{x}_{n}$ is $\hat{x}_{n}$ or $\tilde{x}_{n}$. Typical time courses of absolute estimate errors of instantaneous phases and amplitudes are shown in Figs. 4(a) and 5(a), respectively. Figures 4(b) and 5(b) show the mean absolute estimate errors of instantaneous phases and amplitudes averaged over all the trials, respectively. Both of the mean absolute estimate errors of instantaneous phases and amplitudes estimated by the MRFPA were less than those estimated by the Hilbert transform (Wilcoxon signed rank test, $p<0.0001)$. This result indicates that the highly accurate estimates of instantaneous phases and amplitudes can be obtained by using the MRFPA.

\section{B. EEG experiments}

It is known that there is a relationship between alpha rhythm phase at a flash stimulus onset and RT [7]. Therefore, to investigate practical usefulness of the MRFPA for experimental EEG data, we examined whether the relationship be- 

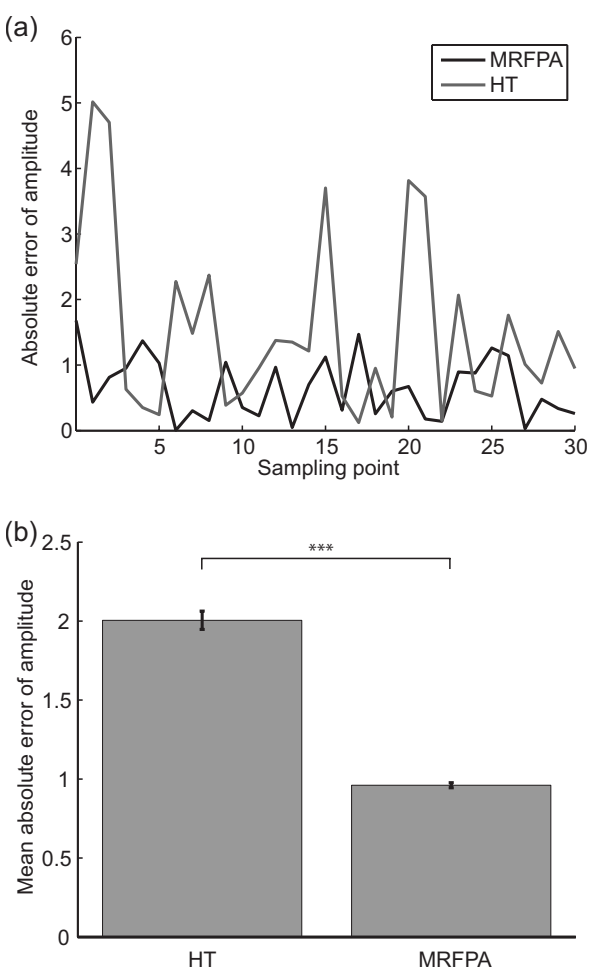

FIG. 5. (a) Typical time courses of absolute estimate error of instantaneous amplitude estimated by the MRFPA (black line) and the Hilbert transform (gray line). (b) Mean absolute estimate errors of instantaneous amplitudes. Values averaged over the trials are illustrated. The error bars indicate the standard errors. $* * *$ indicates $p<0.0001$.

tween the alpha phases at a flash stimulus onset and RTs emerges more clearly by using the MRFPA than the Hilbert transform.

EEG data of six clinically normal adult volunteers with their eyes closed were recorded. None of the subjects had any history of relevant neurological or visual disorders. All subjects gave written informed consent. The study received ethical approval from the Ethics Committee for Human and Animal Research at the Graduate School of Frontier Sciences, The University of Tokyo. The flash stimuli were projected onto a screen (visual angle: $50^{\circ} \times 60^{\circ}$, luminance: $730 \mathrm{~cd} / \mathrm{m}^{2}$ ). Their duration was $1 / 60 \mathrm{~s}$ and interstimulus interval was $2-4 \mathrm{~s}$. The number of trials was 500 . The subjects were asked to press the button with their right index finger as soon as they perceived a flash. To exclude the effects of outliers, we calculated the mean of RT over all the trials and used trials with RTs within the mean $\pm 100 \mathrm{~ms}$ for each subject for the following analysis. EEG data were sampled at $1 \mathrm{kHz}$, digitally band-pass filtered between 5 and $45 \mathrm{~Hz}$, and down sampled to $100 \mathrm{~Hz}$.

In order to estimate the phase of the alpha rhythm at the stimulus onset we used EEG data from -1 to $0.05 \mathrm{~s}(0 \mathrm{~s}$ corresponds to the stimulus onset), since prominent evoked responses by the flash stimulus occurred after $0.05 \mathrm{~s}$. The phase of the alpha rhythm of each trial at the stimulus onset was estimated by the MRFPA and the Hilbert transform. Figure 6 shows typical time courses of estimated amplitude and phase by the MRFPA and the Hilbert transform. The esti-

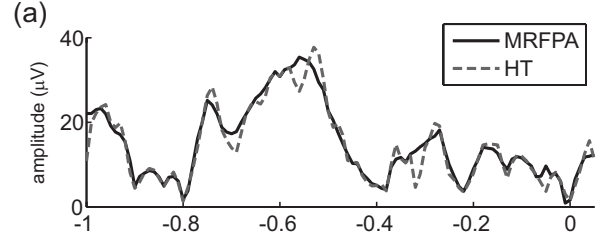

(b)

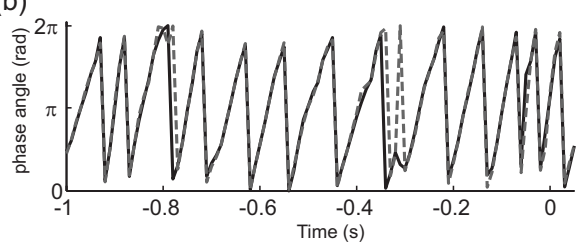

FIG. 6. Typical time courses of estimated amplitude (a) and phase (b) of experimental EEG data by the MRFPA (black line) and the Hilbert transform (gray broken line).

mated time courses were basically similar to each other, though the estimated values varied. The method of the following analysis was similar to that in the previous study [7]. The trials for each subject were sorted based on the alpha rhythm phase at electrode $\mathrm{Pz}$ at the stimulus onset and divided into 10 bins. The $q$ th bin included the trials whose alpha phases at the stimulus onset were within $2 \pi \times(q$ $-1) / 10-2 \pi \times q / 10$. The mean RT for each bin was calculated and the bins whose mean RT were fastest or slowest were selected. Then we examined whether the RTs of the selected bins were significantly different by using a t test.

The results shown in Table I indicate that the number of subjects who showed significant difference was larger when the phases were estimated by the MRFPA than by the Hilbert transform. This result suggests that the relationship between the alpha phases at the flash stimulus onset and the RTs emerges more clearly by using the MRFPA than the Hilbert transform.

\section{DISCUSSION}

In this study, we have modeled the phase and amplitude of the alpha rhythm on an MRF model and then constructed a $\mathrm{BP}$ algorithm that estimates the instantaneous phase and amplitude and calculates the marginal likelihood. Maximizing the marginal likelihood enables us to determine the hyperparameters. We have proved that when the observed data consist of only one frequency signal with constant amplitude and Gaussian noise, the instantaneous phase and amplitude estimated by the modified model from the MRFPA is consistent with that estimated by the Hilbert transform of the filtered signal from the observed data. Thus, we can argue that the MRFPA is an advanced model of the modified model, which is comparable to the Hilbert transform, to suit a priori knowledge about the alpha rhythm.

The likelihood function [Eq. (2)] and the prior distribution of phase [Eq. (3)] include nonlinear terms, and the Laplace approximation is often used in this case. However, the Laplace approximation approximates a marginal posterior distribution by a unimodal Gaussian distribution, and accordingly, it probably gives a poor estimation value since the marginal posterior distribution is not a unimodal distribution 
TABLE I. The influence of the estimation method that is the MRFPA or the Hilbert transform on the relationship between reaction times and the alpha phases at the stimulus onset. * indicates significant difference $(p<0.05)$.

\begin{tabular}{|c|c|c|c|c|c|c|}
\hline \multirow[b]{3}{*}{ Subject No. } & \multicolumn{3}{|c|}{ Estimated by MRFPA } & \multicolumn{3}{|c|}{ Estimated by Hilbert transform } \\
\hline & \multicolumn{2}{|c|}{$\begin{array}{l}\text { Reaction time } \\
(\mathrm{ms})\end{array}$} & \multirow[b]{2}{*}{$p$ value } & \multicolumn{2}{|c|}{$\begin{array}{l}\text { Reaction time } \\
(\mathrm{ms})\end{array}$} & \multirow[b]{2}{*}{$p$ value } \\
\hline & Slowest bin & Fastest bin & & Slowest bin & Fastest bin & \\
\hline 1 & 262.09 & 244.21 & $p=0.00136 *$ & 257.64 & 243.52 & $p=0.0147 *$ \\
\hline 2 & 348.54 & 323.59 & $p=0.0619$ & 344.39 & 326.58 & $p=0.159$ \\
\hline 3 & 259.17 & 248.14 & $p=0.122$ & 255.13 & 248.68 & $p=0.378$ \\
\hline 4 & 202.97 & 192.5 & $p=0.0496 *$ & 203.19 & 193.33 & $p=0.0686$ \\
\hline 5 & 256.56 & 237.47 & $p=0.0187 *$ & 261.3 & 229.33 & $p=0.000417 *$ \\
\hline 6 & 222.64 & 207.12 & $p=0.0131 *$ & 222.52 & 208.83 & $p=0.0719$ \\
\hline
\end{tabular}

in the MRFPA (Fig. 2). By using the BP algorithm, we can calculate the exact marginal posterior distribution, and therefore, the BP algorithm is useful to estimate the instantaneous phase and amplitude in the MRFPA.

Estimations from the simulated data indicate that the observation noise can be reduced more efficiently by the MRFPA than by the MRFS (Fig. 3). In spite of a decrease in the absolute estimate error of the MRFS from the absolute observation error, the amount of the decrease is very small. The estimated hyperparameters of the MRFS suggest a reason, showing that the estimated value of $\hat{\lambda}(0.0336)$, which controls the smoothness of the signal, is much smaller than that of $\hat{\eta}(816)$, which controls the observation noise strength. Since a small value of $\hat{\lambda}$ indicates poor smoothness of the signal change, the MRF model in Eq. (22) with a small value of $\hat{\lambda}$ explains not only the signal changes but also observation noises. The reason why $\hat{\lambda}$ is very small is that the signal of the alpha rhythm exhibits a large periodic change. This result indicates that it is important to adopt the MRFPA when a signal itself changes periodically, as in the case of the alpha rhythm.

Comparison of the estimation accuracy of the instantaneous phase and amplitude indicates that the estimates of the instantaneous phase and amplitude obtained by using the MRFPA are more accurate than those obtained by using the Hilbert transform (Figs. 4 and 5). The Hilbert transform is commonly used to estimate instantaneous phases and amplitudes of the alpha rhythm [13-15]. The fact that it regards all of the observed data, including observation noises, as signals causes the loss of precision of instantaneous phase and amplitude estimation. However, the MRFPA reduces observation noises, and therefore, it achieves higher performance in the instantaneous phase and amplitude estimation.

By using experimental EEG data, we have shown that the relationship between the alpha phases at the flash stimulus onset and RTs emerges more clearly by using the MRFPA than the Hilbert transform (Table I). A low-estimation precision of alpha phase obscures the relationship between RTs and the alpha phase; therefore this result suggests that the MRFPA achieves higher performance in the instantaneous phase estimation for experimental EEG data. The experimental data include noises that are not exactly Gaussian, since experimental data intrinsically include a variety of types of non-Gaussian noise, such as brain noises and environmental noises. At the least, the experimental data used in this study include non-Gaussian noise because of band-pass filtering. Therefore this result also shows robustness of the MRFPA to non-Gaussian noises in experimental EEG data.

Recently, many experimental studies have shown that the phase and amplitude of the alpha rhythm influence not only cognitive task performances but also the perception itself $[3,4,6,8,9,30]$. Since these studies only discuss the results obtained by averaging the values over trials because of the low precision of the instantaneous phase and amplitude estimation by the previous methods, asynchronous neural activities between trials are unknown. Moreover, although it has been suggested that the alpha rhythm should be regarded as signals generated by a nonlinear oscillator or an assembly of coupled nonlinear oscillators $[17,31,32]$, it is still controversial whether an external perturbation, which is a visual stimulus, can influence the phase of the alpha rhythm $[18,19,33-35]$. This is partly due to the low precision of the instantaneous phase estimation by the previous methods. By using the MRFPA, we can estimate the instantaneous phases and amplitudes of the alpha rhythm more precisely, and therefore, the MRFPA can contribute to resolving these problems. Moreover, we can use the MRFPA for other EEG oscillations such as the theta, beta, mu, and gamma rhythms, and it has been suggested that these oscillations and interactions between them play important roles in brain function [36-39]. Thus, we believe that our novel method will contribute to advancing EEG-based studies.

\section{ACKNOWLEDGMENTS}

This study was partially supported by Grant-in-Aid for Scientific Research on Innovative Areas "The study on the neural dynamics for understanding communication in terms of complex hetero systems (Grant No. 4103)" (Grant No. 21120012), Grant-in-Aid for Young Scientists (B) (Grant No. 21700480), Grant-in-Aid for Scientific Research (A) (Grant 
No. 20240020), and Grant-in-Aid for challenging Exploratory Research (Grant No. 22650041) of The Ministry of Education, Culture, Sports, Science, and Technology, Japan.

\section{APPENDIX: ANALYTICAL SOLUTION OF MRFS}

The MRFS can be analytically solved and thus the messages are expressed as

$$
M_{L}^{*}\left(m_{k} \mid \eta, \lambda\right)=A_{L}^{k} \exp \left[-\frac{B_{L}^{k}}{2}\left(m_{k}-C_{L}^{k}\right)^{2}+D_{L}^{k}\right],
$$

and

$$
M_{R}^{*}\left(m_{k} \mid \eta, \lambda\right)=A_{R}^{k} \exp \left[-\frac{B_{R}^{k}}{2}\left(m_{k}-C_{R}^{k}\right)^{2}\right]+D_{R}^{k},
$$

where

$$
\begin{gathered}
A_{L}^{k}=\sqrt{\frac{\eta \lambda}{2 \pi\left(B_{L}^{k-1}+\eta+\lambda\right)},} \\
B_{L}^{k}=\eta \frac{\lambda+B_{L}^{k-1}}{\eta+\lambda+B_{L}^{k-1}},
\end{gathered}
$$

$$
C_{L}^{k}=\frac{\lambda y_{k-1}+B_{L}^{k-1} C_{L}^{k-1}}{\lambda+B_{L}^{k-1}}
$$

$$
D_{L}^{k}=D_{L}^{k-1}-\frac{\lambda B_{L}^{k-1}}{2\left(\lambda+B_{L}^{k-1}\right)}\left(y_{k-1}-C_{L}^{k-1}\right)^{2},
$$

$$
A_{R}^{k}=\sqrt{\frac{\eta \lambda}{2 \pi\left(B_{R}^{k-1}+\eta+\lambda\right)}},
$$

$$
B_{R}^{k}=\eta \frac{\lambda+B_{R}^{k-1}}{\eta+\lambda+B_{R}^{k-1}},
$$

$$
C_{R}^{k}=\frac{\lambda y_{k-1}+B_{R}^{k-1} C_{R}^{k-1}}{\lambda+B_{R}^{k-1}},
$$

and

$$
D_{R}^{k}=D_{R}^{k-1}-\frac{\lambda B_{R}^{k-1}}{2\left(\lambda+B_{R}^{k-1}\right)}\left(y_{k-1}-C_{R}^{k-1}\right)^{2} .
$$

[1] P. A. Robinson, C. J. Rennie, J. J. Wright, H. Bahramali, E. Gordon, and D. L. Rowe, Phys. Rev. E 63, 021903 (2001).

[2] S. C. O'Connor and P. A. Robinson, Phys. Rev. E 70, 011911 (2004).

[3] S. Palva and J. M. Palva, Trends Neurosci. 30, 150 (2007).

[4] W. Klimesch, Int. J. Psychophysiol 24, 61 (1996).

[5] O. Jensen, J. Gelfand, J. Kounios, and J. E. Lisman, Cereb. Cortex 12, 877 (2002).

[6] H. Van Dijk, J. M. Schoffelen, R. Oostenveld, and O. Jensen, J. Neurosci. 28, 1816 (2008).

[7] E. Callaway and C. L. Yeager, Science 132, 1765 (1960).

[8] N. A. Busch, J. Dubois, and R. VanRullen, J. Neurosci. 29, 7869 (2009).

[9] K. E. Mathewson, G. Gratton, M. Fabiani, D. M. Beck, and T. Ro, J. Neurosci. 29, 2725 (2009).

[10] J. R. Wolpaw, N. Birbaumer, D. J. McFarland, G. Pfurtscheller, and T. M. Vaughan, Clin. Neurophysiol. 113, 767 (2002).

[11] J. R. Wolpaw and D. J. McFarland, Proc. Natl. Acad. Sci. U.S.A. 101, 17849 (2004).

[12] J. Mellinger, G. Schalk, C. Braun, H. Preissl, W. Rosenstiel, N. Birbaumer, and A. Kubler, Neuroimage 36, 581 (2007).

[13] A. Matani, Y. Naruse, Y. Terazono, T. Iwasaki, N. Fujimaki, and T. Murata, IEEE Trans. Biomed. Eng. 57, 1117 (2010).

[14] V. V. Nikulin and T. Brismar, Neuroscience 137, 647 (2006).

[15] V. V. Nikulin, K. Linkenkaer-Hansen, G. Nolte, S. Lemm, K. R. Muller, R. J. Ilmoniemi, and G. Curio, Eur. J. Neurosci. 25, 3146 (2007).

[16] J. M. Palva, S. Palva, and K. Kaila, J. Neurosci. 25, 3962 (2005).

[17] Y. Naruse, A. Matani, Y. Miyawaki, and M. Okada, Hum. Brain Mapp 31, 703 (2010).
[18] S. Makeig, M. Westerfield, T. P. Jung, S. Enghoff, J. Townsend, E. Courchesne, and T. J. Sejnowski, Science 295, 690 (2002).

[19] Y. Naruse, A. Matani, T. Hayakawa, and N. Fujimaki, Neuroimage 32, 1221 (2006).

[20] H. Nishimori and K. Y. Michael Wong, Phys. Rev. E 60, 132 (1999).

[21] K. Tanaka, H. Shouno, M. Okada, and D. M. Titterington, J. Phys. A 37, 8675 (2004).

[22] C. M. Bishop, Pattern Recognition and Machine Learning (Spinger-Verlag, Berlin, 2006).

[23] K. Linkenkaer-Hansen, V. V. Nikouline, J. M. Palva, and R. J. Ilmoniemi, J. Neurosci. 21, 1370 (2001).

[24] J. Pearl, Probabilistic Reasoning in Intelligent Systems (Morgan Kaufmann, San Mateo, 1988).

[25] K. Takiyama, K. Katahira, and M. Okada, J. Phys. Soc. Jpn. 78, 064003 (2009).

[26] K. Watanabe, H. Tanaka, K. Miura, and M. Okada, IEICE Trans. Inf. Syst., E92.D, 1362 (2009).

[27] K. Tanaka, J. Phys. A 35, R81 (2002).

[28] H. Nishimori, Bussei Kenkyu 73, 850 (2000).

[29] A. Oppenheim, R. W. Schafer, and J. R. Buck, Discrete-Time Signal Processing (Prentice-Hall, Upper Saddle River, 1999).

[30] F. J. Varela, A. Toro, E. R. John, and E. L. Schwartz, Neuropsychologia 19, 675 (1981).

[31] F. H. Lopes da Silva, J. P. Pijn, D. Velis, and P. C. G. Nijssen, Int. J. Psychophysiol 26, 237 (1997).

[32] C. J. Stam, J. P. M. Pijn, P. Suffczynski, and F. H. Lopes da Silva, Clin. Neurophysiol. 110, 1801 (1999).

[33] A. Mazaheri and O. Jensen, Proc. Natl. Acad. Sci. U.S.A. 103, 2948 (2006).

[34] M. L. Risner, C. J. Aura, J. E. Black, and T. J. Gawne, Neu- 
roimage 45, 463 (2009).

[35] W. Klimesch, P. Sauseng, and W. Gruber, Neuroimage 47, 5 (2009).

[36] C. Tallon-Baudry, O. Bertrand, C. Delpuech, and J. Permier, J. Neurosci. 17, 722 (1997).

[37] H. Mizuhara and Y. Yamaguchi, Neuroimage 36, 232 (2007).
[38] P. Tass, M. G. Rosenblum, J. Weule, J. Kurths, A. Pikovsky, J. Volkmann, A. Schnitzler, and H. J. Freund, Phys. Rev. Lett. 81, 3291 (1998).

[39] P. A. Tass, T. Fieseler, J. Dammers, K. Dolan, P. Morosan, M. Majtanik, F. Boers, A. Muren, K. Zilles, and G. R. Fink, Phys. Rev. Lett. 90, 088101 (2003). 